\title{
Influence of Filtration on the Agglomeration Degree of Two Crystalline Amino Acids
}

\author{
Lisa Löbnitz*, Marius Böser, and Hermann Nirschl
}

DOI: $10.1002 /$ cite. 201700110

\begin{abstract}
Separating crystalline material from the mother liquor is often performed by filtration, whereas different options to generate the driving force are possible to use. However, the separation process can affect the structure of crystalline material, either by breakage events or agglomeration. In this work, a method of automated image analysis was used to evaluate the agglomeration degree of two crystalline amino acids after a pressure or vacuum filtration. The information resulting from this consideration enables to adjust the crystal structure depending on the individual filtration type.
\end{abstract}

Keywords: Agglomeration degree, Crystalline amino acids, Filtration

Received: August 25, 2017; accepted: January 09, 2018

\section{Introduction}

The production of fine chemicals and pharmaceuticals often includes fermentation. Due to the high amount of impurities the final molecule can be obtained in a solid, pure and stable form under great effort. For this purpose, crystallization is widely used due to its selectivity. By applying the appropriate crystallization conditions, the end product is of high purity and stability. This method is also used in the production of therapeutically proteins because of the resulting reduction of complex chromatography steps $[1,2]$.

Besides the crystallization the production process consists of the downstream operations separation, drying and optionally milling or further formulation. The aim of this process is a product with well-defined properties concerning particle size, size distribution, flow and surface characteristics $[2,3]$. Adjusting the required formulation of the particles during the production is one of the most challenging parts in the process. During the various process steps the outer appearance of the crystals can be changed and the desired particle properties cannot be reached. These changes can result during crystallization [3-8]. However, also the following operations have a considerable impact on crystal properties. For instance, breakage or agglomeration due to residual mother liquor containing solved material adhering to the crystals can occur.

Especially agglomeration has a high impact on further formulation and the efficiency of pharmaceutical ingredients [9]. In some cases agglomerated material shows a better dissolution and, therefore, a better bioavailability than single crystals $[8,10]$. Furthermore, agglomeration increases the particle size, so that flowability of the powder is improved and undesired events as tablet capping in the solid dosage form can be reduced [11]. Especially the solubility of poor soluble pharmaceuticals is increased by a high specific surface and, hence, a small particle size results, which leads to poor tableting properties. This effect can be improved by agglomeration $[2,12]$.

This work focuses on the agglomeration of crystalline material during the separation step. To analyze the influence of different separation methods on the agglomeration, crystalline amino acids as model substance for active pharmaceutical ingredients are investigated. In addition, the specific characteristics of the product systems are considered by examining different types of amino acids. To characterize the influence of different process parameters on the agglomeration of single crystals, a method has to be used allowing a differentiation between single and agglomerated crystals. Current research proposes different approaches to characterize and classify particles regarding their outer appearance [13-16]. For example, microscopy particle characterization followed by a principle component analysis was executed $[8,17]$. Automated classification of particles was performed by using discriminant factorial analysis (DFA) [9, 18-21]. Besides this multivariate data analysis method, machine learning algorithms can be applied to classify particles. State-of-the art algorithms for different classification problems are support vector machine (SVM), decision tree, random forest and deep learning [22]. In this work two existing methods $[13,14]$ with orthogonal properties were used and evolved to meet the specific requirements of the particle system.

Lisa Löbnitz, Marius Böser, Prof. Dr.-Ing. Hermann Nirschl lisa.loebnitz@kit.edu

Karlsruhe Institute of Technology, Institute of Mechanical Process Engineering and Mechanics, Straße am Forum 8, 76131 Karlsruhe, Germany. 


\section{Material and Methods}

\subsection{Particle System}

Two different types of amino acids were used. Both particle systems were obtained in crystalline form: $L$-glutamic acid ( $L$-Glu, Sigma Aldrich Co. LLC, $99 \%$ ) and $L$-phenylalanine ( $L$-Phe, Carl Roth GmbH + Co. KG, Cellpure, >99\%). Crystal suspension ( $10 \%$ by mass) was prepared by dispersing crystals in saturated solution of its amino acid type. Therefore, a saturated solution was prepared by solving the proper amount of amino acid to reach the saturation concentration at $20^{\circ} \mathrm{C}$. The solution was equilibrated for $24 \mathrm{~h}$ at constant saturation temperature. Following, the solution was supersaturated with amino acid crystals to obtain a crystal suspension with the desired mass concentration. A particle size and agglomeration degree distribution was prepared by the method described in Sect. 2.3.

\subsection{Filtration Experiments}

After preparing and evenly distributing the crystal suspension in 50-g vessels, the samples were filtered. Therefore, either a vacuum filtration plate with a filtration area of $44.5 \mathrm{~cm}^{2}$ or a pressure Nutsche instrument (BHS Sonthofen $\mathrm{GmbH}$ ) with a filtration area of $19.56 \mathrm{~cm}^{2}$ was used. Additional parameter describing the filtration behavior of crystalline $L$-Glu can be found in related work $[23,24]$. Filtration devices that are using a pressure filtration can be operated up to $25 \cdot 10^{5} \mathrm{~Pa}$ [25]. To realize process-related conditions regarding the separation of material with medium filtration resistance, the considered pressure difference during the filtration is $3 \cdot 10^{5} \mathrm{~Pa}$. Hence, a medium mechanical stress situation is performed and can be compared to a vacuum filtration at $0.8 \cdot 10^{5} \mathrm{~Pa}$ pressure difference, which is usually used in continuous vacuum filtration devices.

The wet filter cake was analyzed regarding the particle size and agglomeration degree distribution. Therefore, a sample of the filter cake was dispersed in saturated solution and analyzed. Subsequently, the filter cake was dried by means of contact drying at $45^{\circ} \mathrm{C}$ for two days. This method of drying was chosen because the influence of the filtration on particle appearance and not the drying method itself should be investigated in this work. The dried filter cake was suspended in saturated solution and analysis of particle size and agglomeration degree was performed. This method was used in the case of $L$-Glu, which has a small solubility and temperature dependency. In contrast, no wet cake samples of $L$-Phe were made due to the temperature dependency of the solubility of the system.

\subsection{Image and Particle Analysis}

To evaluate the influence of the filtration on crystal agglomeration an automated image analysis was used. This method is based on the work of Terdenge et al. [13], which applies DFA to solve the classification problem, and was evolved by the methods of Ochsenbein et al. [14], which uses a support vector machine as learning algorithm. Both methods use particle descriptors, specifying either the appearance of the particles, e.g., extension or size, or the intensity, e.g., gray values. These parameters are obtained by taking optical images of the crystal suspension by means of a transmitted light microscope (Ortophlan, Leitz Park $\mathrm{GmbH}$ ) with $3.2 \mathrm{x}$ enlargement. Due to the resolution limit of the microscope, particles $<10 \mu \mathrm{m}$ were not considered. After enhancing the contrast and reducing the background interference, the particles were analyzed by the open source software ImageJ. Thereby, the image descriptors can be quantified. Additionally, this method provides a cumulative particle size distribution (PSD) by relating the area of a particle fraction in a defined size range to the total amount of particles.

Previous to the classification of an unknown crystal sample, the classification algorithm must be trained to enable a correlation between image descriptor and classification group. Therefore, a training set for $L$-Glu and $L$-Phe was prepared by manually matching a particle either to the group agglomerates or single particles. In contrast to literature [13] an additional group of waste particles was not chosen. This is because preliminary results did not show any improvement by using an additional classification group. Also, the images did not show any foreign particles, so that a waste group was not needed. The training set values of different image descriptors and their combinations can be related to the classes by classification algorithms.

The considered classification algorithms rely on different methods. The DFA is a linear classification method and separates the classes in the two-dimensional space [26]. Here, a linear combination of the product of the image descriptors and their loadings is computed to describe both, the group agglomerates and single particles. A particle with unknown appearance is described by its image descriptors and a discriminant value is computed with the specific parameter. The particle is assigned to the classes to which the discriminant value is closest [27]. To make the method more sensitive, a weighting factor was implemented. This factor weights image descriptors located near the critical value low, and weights factors whose distance is more exceeded strongly.

While DFA uses a linear classification method, SVMs can perform nonlinear classification [26]. This is because SVM transforms the problem and its solution to a higher space where a separating hyperplane is computed. Transforming the hyperplane back to the original search space can result in a nonlinear division of this space [28]. A detailed look at the methods and algorithm can be found elsewhere [29] and will not be further discussed at this point. The classification method enables grouping particle samples in the 
classes agglomerates and single particles. To describe the amount of agglomerates compared to the total number of particles the description of the agglomeration degree $\operatorname{Ag}(x)$ is used, which is known from literature $[13,18,20]$ and defined as follows:

$\operatorname{Ag}_{\mathrm{j}}(x)=\frac{n_{\text {agglomerates }, \mathrm{j}}}{n_{\text {crystals } \mathrm{j}}}$

The amount of agglomerates $n_{\text {agglomerates,j }}$ in the size range $j$ is placed into relation to the total amount of crystals $n_{\text {crystals,j }}$ in the size range $\mathrm{j}$.

Three key requirements have to be met to evaluate the efficiency of the classification (see Tab.1). They are mainly described by the performance index PI [13], which relates the amount of correctly classified particles $n_{\text {correct }}$ to the total amount of particles $n_{\text {total }}$ :

$\mathrm{PI}=\frac{n_{\text {correct }}}{n_{\text {total }}} \cdot 100 \%$

Table 1. Key requirements for the selection of the classification methods.

\begin{tabular}{ll}
\hline Key requirement & Value \\
\hline Hit accuracy of the training set & $\mathrm{PI}_{\text {total }}>92 \%$ \\
$\begin{array}{l}\text { Equal hit accuracy between the different } \\
\text { classification groups }\end{array}$ & $\mathrm{PI}_{\mathrm{SC}}>90 \% \wedge \mathrm{PI}_{\mathrm{A}}>90 \%$ \\
Hit accuracy of the validation & $\mathrm{PI}_{\text {Val }}>88 \%$ \\
\hline
\end{tabular}

Additionally, the difference between the manually $\left(\mathrm{Ag}_{\text {manual }}\right)$ and automatically $\left(\mathrm{Ag}_{\text {automatic }}\right)$ classified agglomeration degrees is compared

$\Delta \mathrm{Ag}=\left(\left|\mathrm{Ag}_{\text {automatic }}-\mathrm{Ag}_{\text {manual }}\right|\right)$

and the average deviation of $\Delta \mathrm{Ag}$ over the full-size range was minimized to choose the most suitable classification method.

\section{Results and Discussion}

\subsection{Classification and Image Analysis}

In a first step, DFA and SVM were used to find the most suitable classification method. A manually classified database was generated and compared with the results of the different methods. Also, the amount of considered image descriptors was varied to find the optimal solution of the classification method. Fig. 1 shows the crystalline particles of $L$-Glu and $L$-Phe and their manually mapping to agglomerated and single crystals.

Substantial differences between the particle systems can be observed. Whereas $L$-Glu shows sharp edges and clear defined concave areas, $L$-Phe crystals appear spherical with

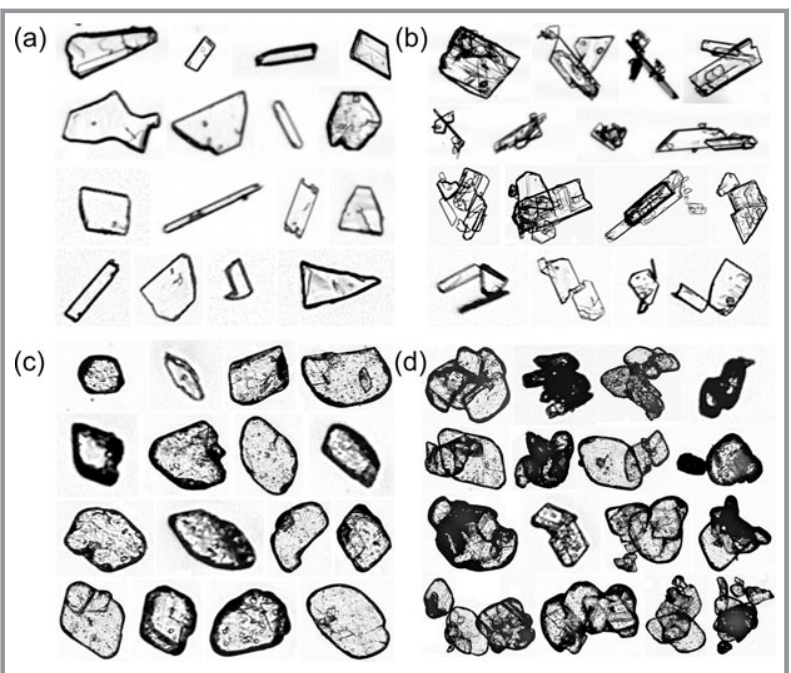

Figure 1. Microscopic pictures of the manually classified crystals of a) single L-Glu crystals, b) agglomerated L-Glu crystals, c) single $L$-Phe crystals, and d) agglomerated $L$-Phe crystals.

a higher amount of opaqueness. Both systems are flat, which eases a comparison between the system due to the similar crystal habitus.

Different combinations of image descriptors were investigated, whereas in the case of $L$-Glu only three combinations met the key requirements summarized in Tab. 1. The comparison between the manually and automatically classified samples of $L$-Glu is shown in Fig. 2. Fig. 2a presents the agglomeration degree of a $L$-Glu batch by means of manual classification by a human expert, a DFA analysis with 9 image descriptors, and two SVM methods with either 4 or 11 image descriptors.

All classification methods match the progression of the manual classification well. To decide which method is the most suitable, the differences between the manually and automatically classified agglomeration degrees were computed (Fig. 2b). Based on the minimal average deviation of the differences of $\Delta \mathrm{Ag}$ in all size ranges of 0.035 , the SVM method with 11 image descriptors is used to characterize $L$-Glu crystals.

Besides this validation with manually classified particles, the result of the characterization by the automated image analysis on particles with slightly different shapes is evaluated. Therefore, $L$-Glu crystals are suspended in an undersaturated solution. As a result, the crystal edges dissolve partially, hence, the crystal shape changes. Fig. 3 shows the results of the particle size distribution and the agglomeration degree. Both curves show changes in their progression between the suspension of crystals in saturated solution and undersaturated solution. The PSD shows a decrease in particle size. Regarding the agglomeration degree $(\mathrm{AgD})$ more information about the process resulting in the reduction of the particle size can be noted. The change in particle shape leads to an increased amount of crystals that are classified as agglomerates. This can be explained by the choice of 

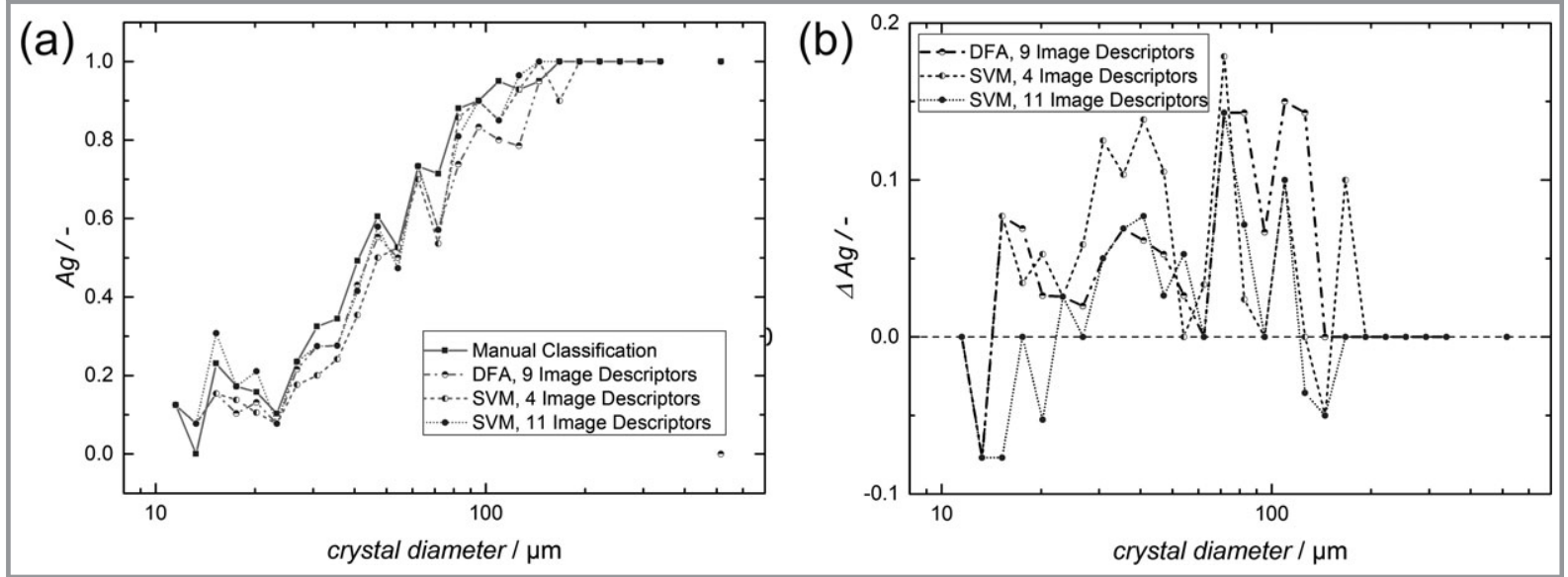

Figure 2. Evaluation of the appropriate classification method of L-Glu by comparison of the a) agglomeration degree of manually and automatically classified crystals, and b) the absolute difference between them.

image descriptors. $L$-Glu crystals are mainly described by form descriptors. When changing the outer appearance of the crystals by dissolving the particle edges, more crystals are classified as agglomerates. Hence, the classification of the particles can be applied to the real crystal systems because of the notification of shape changes.

The process of finding the optimal classification method for $L$-Phe is identical to $L$-Glu, but results in a different method. As most suitable method a DFA classification with 9 image descriptors and a weighting factor was used. Tab. 2 summarizes the most important parameters for identifying the classification method for both particle systems.

The differences between the two particle systems regarding the classification method can be explained by the specific appearance of the crystals. $L$-Glu is more difficult to classify compared to $L$-Phe due to the wide range of outward forms. Whereas single crystals of the latter can be described in an appropriate manner by form parameters, e.g., circularity, crystals of $L$-Glu cannot be classified only by these descriptors, because of the uncertain crystal structure. This is also the reason for SVMs being the more suitable method for the classification. In the case of $L$-Phe the classification problem can be solved in a two-dimensional space by using a linear solution, whereas the separation between the two groups of $L$-Glu needs the solution of a nonlinear problem. This can be achieved more successfully by using SVMs.

\subsection{Influence of the Filtration Method}

The type of filtration has a significant influence on the particle system. Therefore, two methods of filtration are compared regarding the agglomeration degree. The investigations were performed with $L$-Glu. Due to better clarity, the mean values of the measurements and, hence, the principal tendencies are shown in the following diagrams, whereas the deviations are not presented. Fig. 4 shows the PSD and the $\mathrm{AgD}$ of the particles before and after vacuum filtration at a pressure difference of $0.8 \cdot 10^{5} \mathrm{~Pa}$. Also, the differences between the wet and dry filter cakes are compared. The
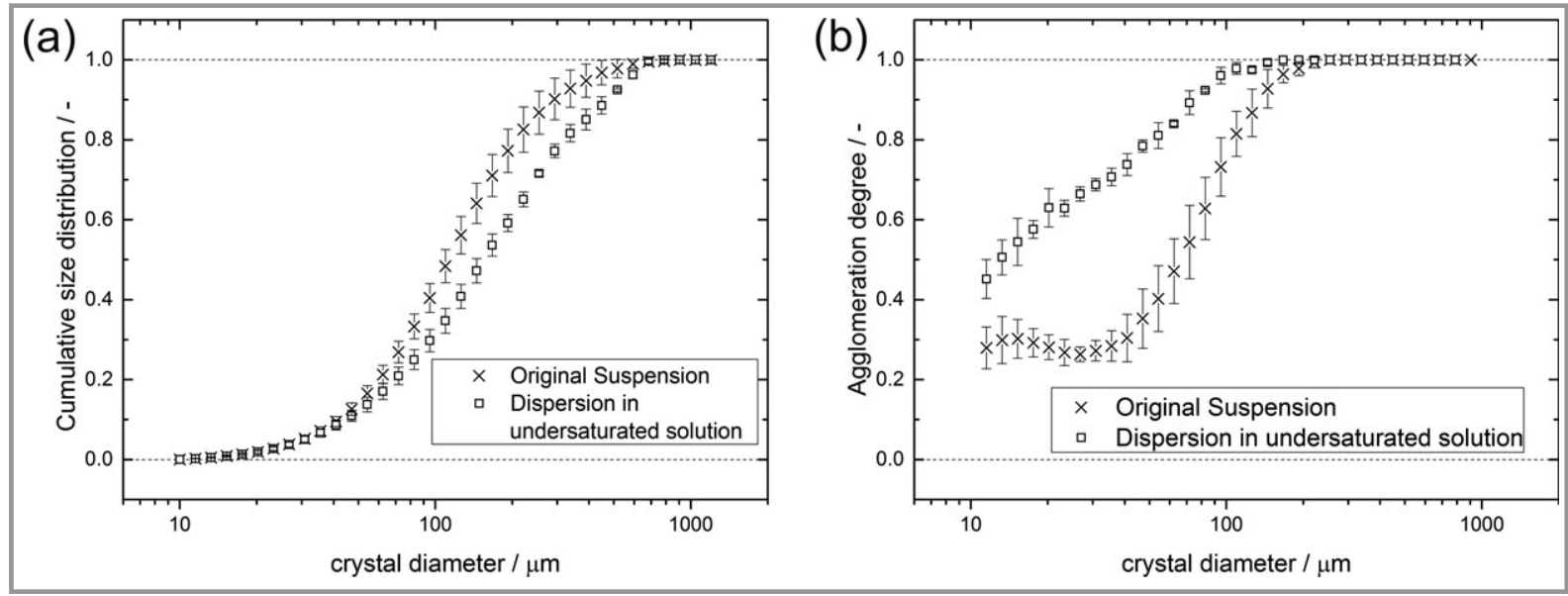

Figure 3. PSD (a) and AgD (b) of an original crystal suspension of L-Glu and a dispersion in undersaturated solution. 
Table 2. Classification methods and parameters for different amino acid systems.

\begin{tabular}{|c|c|c|c|}
\hline & & $\beta$ - $L$-glutamic acid & $L$-phenylalanine \\
\hline \multirow[t]{3}{*}{ Training set } & Number of single particles [-] & 561 & 250 \\
\hline & Number of agglomerates [-] & 537 & 251 \\
\hline & Total number of crystals [-] & 1098 & 501 \\
\hline \multirow[t]{16}{*}{ Most accurate classification } & Method & SVM & DFA \\
\hline & Image descriptors & - particle diameter & - particle diameter \\
\hline & & - area fraction & - area fraction \\
\hline & & - elongation & - elongation \\
\hline & & - solidity & - solidity \\
\hline & & - average grey value & - average grey value \\
\hline & & - deviation of gray values & - circularity \\
\hline & & - roundness & - particle perimeter \\
\hline & & - circularity & \\
\hline & & - area ratio & \\
\hline & & - particle perimeter & \\
\hline & & - modified Waddell sphericity & \\
\hline & $\mathrm{PI}_{\text {total }}[\%]$ & 95.40 & 95.00 \\
\hline & $\mathrm{PI}_{\mathrm{SC}}[\%]$ & 96.10 & 96.40 \\
\hline & $\mathrm{PI}_{\mathrm{A}}[\%]$ & 94.60 & 93.60 \\
\hline & $\mathrm{PI}_{\text {Val }}[\%]$ & 91.75 & 95.00 \\
\hline
\end{tabular}
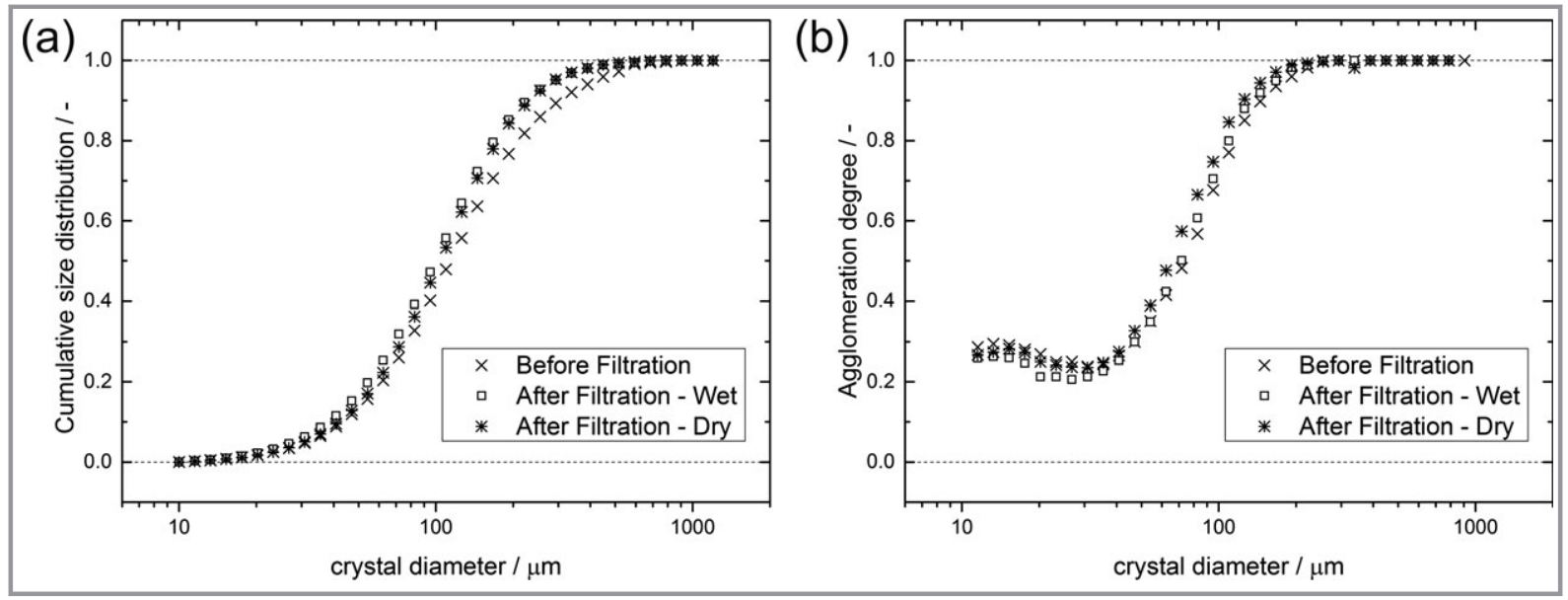

Figure 4. PSD (a) and AgD (b) of $L$-Glu before and after vacuum filtration and drying.

PSD does not change significantly during the filtration process (Fig. 4a). The same observation was made for the AgD (see Fig. 4b). This leads to the conclusion that vacuum filtration has no effect on the agglomeration behavior of $L$-Glu. This is explainable by the pressure difference as well as the solubility of $L$-Glu. Neither breakage events occur during the filtration process nor does agglomeration of fine particles take place. Another cause for changes in agglomeration state is the formation of solid bridges between the single particles because of solved material in the remaining mother liquor. The solubility of $L$-Glu is $0.008 \mathrm{~g}_{\mathrm{As}} \mathrm{g}_{\mathrm{w}}{ }^{-1}$, hence, only a small amount of solid material participates in the formation of solid bridges.
While the vacuum filtration does not show any effect on the agglomeration of crystalline $L$-Glu, different observation can be made for the pressure filtration of $L$-Glu at a pressure difference of $3 \cdot 10^{5} \mathrm{~Pa}$. Fig. 5 shows the results. After the filtration, the PSD (Fig. 5a) of the wet filter cake in the size range of the coarse material shifts to smaller particle sizes. This can be explained by a grinding effect of the pressure on the particle network. No significant changes in this size range can be found for the $\mathrm{AgD}$ (Fig. 5b). The broken crystals, which can be observed in the PSD, are agglomerates. The resulting fragments are also agglomerates, hence, the change cannot be observed in the AgD. 

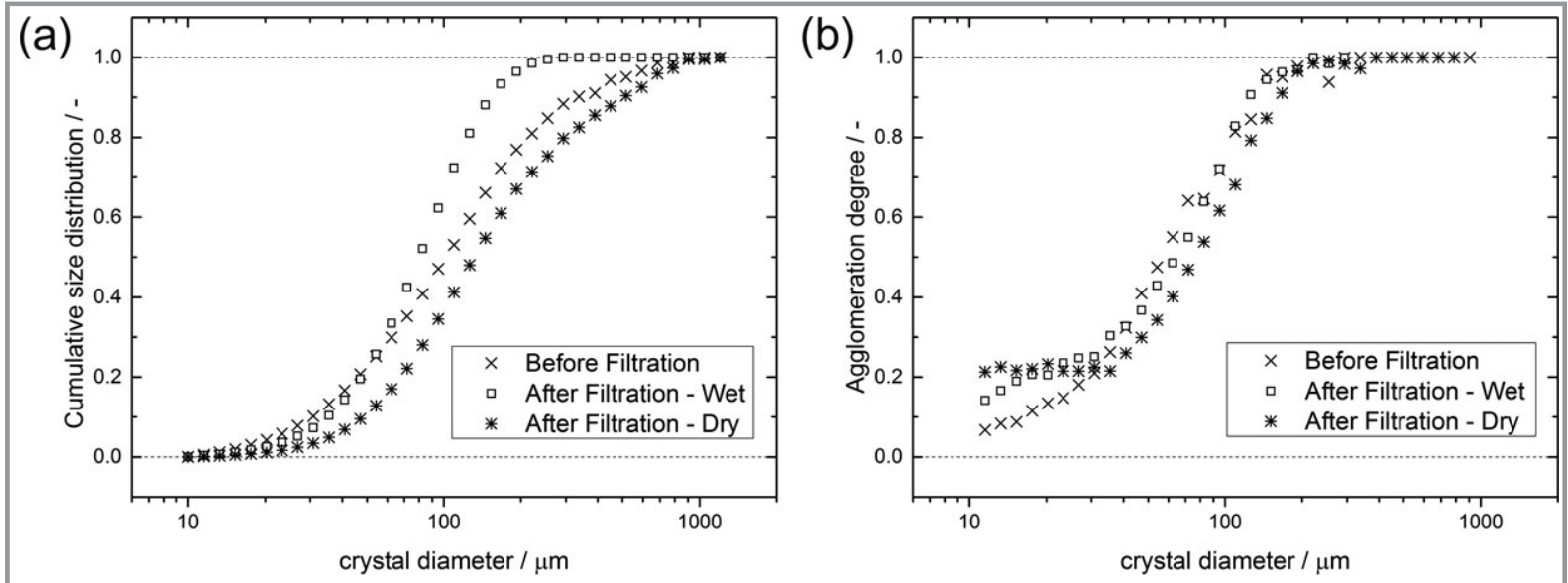

Figure 5. PSD (a) and AgD (b) of $L$-Glu before and after pressure filtration and drying.

In the size range of the fine crystals a different effect can be observed. Whereas no significant changes in the PSD are shown, the AgD increases. This can be attributed to the pressure on the particle network. Fine particles approach each other due to the mechanical stress. Agglomerates are formed because of their surface-to-volume ratio or rather the ratio of adhesive forces to weight force. When looking at particles of the dried filter cake another change in PSD and $\mathrm{AgD}$ can be observed. The particle size decreases compared to the original crystal suspension as well as the wet filter cake. This is explainable by the formation of solid bridges between the particles. Compared to the vacuum filtration the amount of fine crystals is higher. Therefore, the solved material in the mother liquor between the particles is sufficient for agglomeration. This effect can also be seen in the $\mathrm{AgD}$. The amount of agglomerates in the fine particle is increased due to drying.

Summarizing, pressure filtration has a greater effect on the particle shape and the tendency to form agglomerates compared to vacuum filtration. Consequently, vacuum fil- tration shall be used when agglomeration events are not the aim of the production. An increase in particle size can be obtained by using pressure filtration, as well as a reduction of the number of fine particles.

\subsection{Influence of the Particle System}

Besides the investigation of the influence of the filtration type, the reactions of different particle systems to vacuum filtration have been observed. Therefore, $L$-Phe was analyzed before and after vacuum filtration. Additionally, the influence of washing with ethanol was evaluated. This section does not focus on the influence of the particle shape, but of the solubility. L-Phe has a four times higher solubility as $L$-Glu. Therefore, the effect of solid bridges between the single particles is expected to be stronger. This hypothesis can be confirmed by considering the result of the filtration experiments in Fig. 6. Whereas the PSD (Fig. 6a) does not seem to change during the process, the AgD shows a signifi-
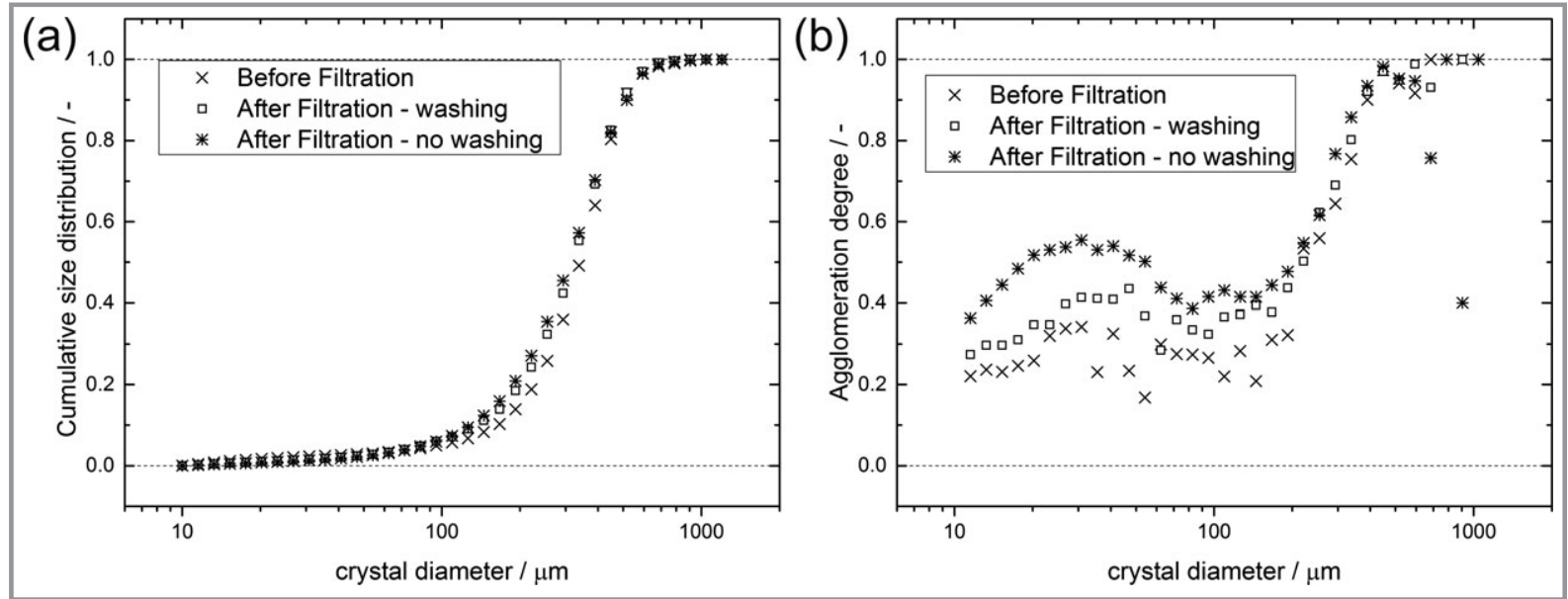

Figure 6. PSD (a) and AgD (b) of L-Phe before and after vacuum filtration and ethanol washing. 
cant reaction. This situation illustrates the importance of not only looking at the PSD, but also at the individual shape and appearance of the particles.

The dried filter cake contains more agglomerates in the size range between 10 to $150 \mu \mathrm{m}$, whereas the coarse particle fraction does not change. This leads to the conclusion that fine particles agglomerate due to the solid material in the mother liquor and subsequently form solid bridges. This hypothesis can be reinforced by the washing results. When washing the filter cake with ethanol the amount of agglomerates compared to the unwashed filter cake reduces. This can be explained by the replacement of the mother liquor in between the pore system with ethanol. Therefore, no additional solid bridges based on crystalline material can be formed and the agglomeration events can be reduced.

\section{Conclusion}

In this work, the influence of the solid-liquid separation using the filtration as physical process on the agglomeration behavior of crystalline amino acids was investigated. Two main aspects were considered. On the one hand, the filtration type, either pressure or vacuum filtration, was analyzed to have a closer look at the impact of the chosen process type. With regard to the formulation following the isolation of the target molecule in its solid state, the selection of the appropriate separation step can be supported. $L$-Glu showed no reaction concerning the agglomeration when using the vacuum filtration, whereas the pressure filtration led to an increasing amount of agglomerates. On the other hand, the influence of the particle system, in particular the solubility of the crystalline material, on the agglomeration process was of interest. Whereas $L$-Glu did not show any tendency to agglomerate because of the solved material in the mother liquor remaining in the pore system, $L$-Phe crystals agglomerated due to this effect. The agglomeration could be reduced by washing the filter cake. Hence, the tendency to agglomerate does not only depend on the used separation strategy but also on the particle system itself. A detailed look at the specific particle properties as well at the most suitable separation technology is required to guarantee an optimal separation process and particle engineering.

\section{Symbols used}

$\begin{array}{lll}\text { Ag } & {[-]} & \text { agglomeration degree } \\ n & {[-]} & \text { number of particles } \\ \text { PI } & {[\%]} & \text { performance index } \\ \Delta \mathrm{Ag} & {[-]} & \text { difference of agglomeration degree }\end{array}$

\section{Subscripts}

A agglomerates

J size class
SC
single crystals
total total amount of particles
Val validation

\section{Abbreviations}

AgD agglomeration degree

DFA discriminant factorial analysis

L-Glu $\quad L$-glutamic acid

$L$-Phe $\quad L$-phenylalanine

PSD particle size distribution

SVM support vector machine

\section{References}

[1] S. Schmidt, D. Havekost, K. Kaiser, J. Kauling, H.-J. Henzler, Eng. Life Sci. 2005, 5 (3), 273 - 276. DOI: 10.1002/elsc.200500116

[2] S. Kim, B. Lotz, M. Lindrud, K. Girard, T. Moore, K. Nagarajan, M. Alvarez, T. Lee, F. Nikfar, M. Davidovich, S. Srivastava, S. Kiang, Org. Process Res. Dev. 2005, 9 (6), 894 - 901. DOI: 10.1021/op050091q

[3] D. Wieckhusen, Chimia 2006, 60 (9), 598 - 604. DOI: 10.2533/ chimia. 2006.598

[4] S. Kim, C. Wei, S. Kiang, Org. Process Res. Dev. 2003, 7 (6), 997 - 1001. DOI: 10.1021/op034107t

[5] N. S. Tavare, V. Patwardhan, AIChE J. 1992, 38 (3), 377 - 384. DOI: 10.1002/aic.690380306

[6] R. C. Zumstein, R. W. Rousseau, Chem. Eng. Sci. 1989, 44 (10), 2149 - 2155. DOI: 10.1016/0009-2509(89)85149-8

[7] M. Fujiwara, P. S. Chow, D. L. Ma, R. D. Braatz, Cryst. Growth Des. 2002, 2 (5), 363 - 370. DOI: 10.1021/cg0200098

[8] E. M. Ålander, M. S. Uusi-Penttilä, Å. C. Rasmuson, Ind. Eng. Chem. Res. 2004, 43 (2), 629 - 637. DOI: 10.1021/ie0301280

[9] M.-N. Pons, H. Vivier, J. Dodds, Part. Part. Syst. Charact. 1997, 14 (6), 272 - 277. DOI: 10.1002/ppsc. 19970140603

[10] M. Jbilou, A. Ettabia, A. M. Guyot-Hermann, J. C. Guyot, Drug Dev. Ind. Pharm. 1999, 25 (3), 297 - 305. DOI: 10.1081/DDC100102174

[11] N. Blagden, M. de Matas, P. T. Gavan, P. York, Adv. Drug Delivery Rev. 2007, 59 (7), 617 - 630. DOI: 10.1016/j.addr.2007.05.011

[12] N. Rasenack, B. W. Müller, Int. J. Pharm. 2002, 244 (1 - 2), 45 - 57. DOI: 10.1016/S0378-5173(02)00296-X

[13] L.-M. Terdenge, S. Heisel, G. Schembecker, K. Wohlgemuth, Chem. Eng. Sci. 2015, 133, 157 - 169. DOI: 10.1016/ j.ces.2015.03.002

[14] D. R. Ochsenbein, T. Vetter, S. Schorsch, M. Morari, M. Mazzotti, Cryst. Growth Des. 2015, 15 (4), 1923 - 1933. DOI: 10.1021/ acs.cgd.5b00094

[15] V. Kofler, U. Riebel, F. Löffler, Chem. Ing. Tech. 1991, 63 (2), 140 - 142. DOI: $10.1002 /$ cite. 330630216

[16] F. Ros, S. Guillaume, G. Rabatel, F. Sevila, Food Control 1995, 6 (1), 37 - 43. DOI: 10.1016/0956-7135(95)91452-Q

[17] E. M. Ålander, M. S. Uusi-Penttilä, Å. C. Rasmuson, Powder Technol. 2003, 130 (1 - 3), 298 - 306. DOI: 10.1016/S00325910(02)00208-5

[18] N. Faria, M. N. Pons, S. Feyo de Azevedo, F. A. Rocha, H. Vivier, Powder Technol. 2003, 133 (1 - 3), 54 - 67. DOI: 10.1016/S00325910(03)00078-0

[19] Z. Q. Yu, P. S. Chow, R. B. Tan, Chem. Eng. Sci. 2007, 62 (14), 3777 - 3786. DOI: $10.1016 /$ j.ces.2007.04.002 
[20] Z. Q. Yu, R. Tan, P. S. Chow, J. Cryst. Growth 2005, 279 (3 - 4), 477 - 488. DOI: $10.1016 /$ j.jcrysgro.2005.02.050

[21] A. Ferreira, N. Faria, F. Rocha, S. Feyo de Azevedo, A. Lopes, Chem. Eng. Res. Des. 2005, 83 (4), 331 - 338. DOI: 10.1205/ cherd.04145

[22] S. Suthaharan, Machine Learning Models and Algorithms for Big Data Classification, Vol. 36, Springer US, Boston, MA 2016

[23] L. Löbnitz, J. Singer, H. Anlauf, H. Nirschl, Chem. Eng. Technol. 2017, 17 (4), 684. DOI: 10.1002/ceat.201600527

[24] R. Beck, A. Häkkinen, D. Malthe-Sørenssen, J.-P. Andreassen, Sep. Purif. Technol. 2009, 66 (3), 549 - 558. DOI: 10.1016/j.seppur.2009.01.018

[25] W. Müller, Mechanische Verfahrenstechnik und ihre Gesetzmäßigkeiten, 2nd ed., Oldenbourg, München 2014.

[26] T. Hastie, R. Tibshirani, J. Friedman, The Elements of Statistical Learning, 2nd ed., Springer, New York 2009.

[27] A. C. Rencher, Methods of multivariate analysis, 2nd ed., Wiley Series in Probability and Mathematical Statistics, John Wiley \& Sons, New York 2002.

[28] M. A. Hearst, S. T. Dumais, E. Osuna, J. Platt, B. Scholkopf, IEEE Intell. Syst. Their Appl. 1998, 13 (4), 18 - 28. DOI: 10.1109/ 5254.708428

[29] K. Backhaus, B. Erichson, W. Plinke, R. Weiber, Multivariate Analysemethoden, Springer, Berlin 2016.

\section{ACHEMA 2018}

\section{1 - 15 June 2018 \\ Frankfurt am Main}

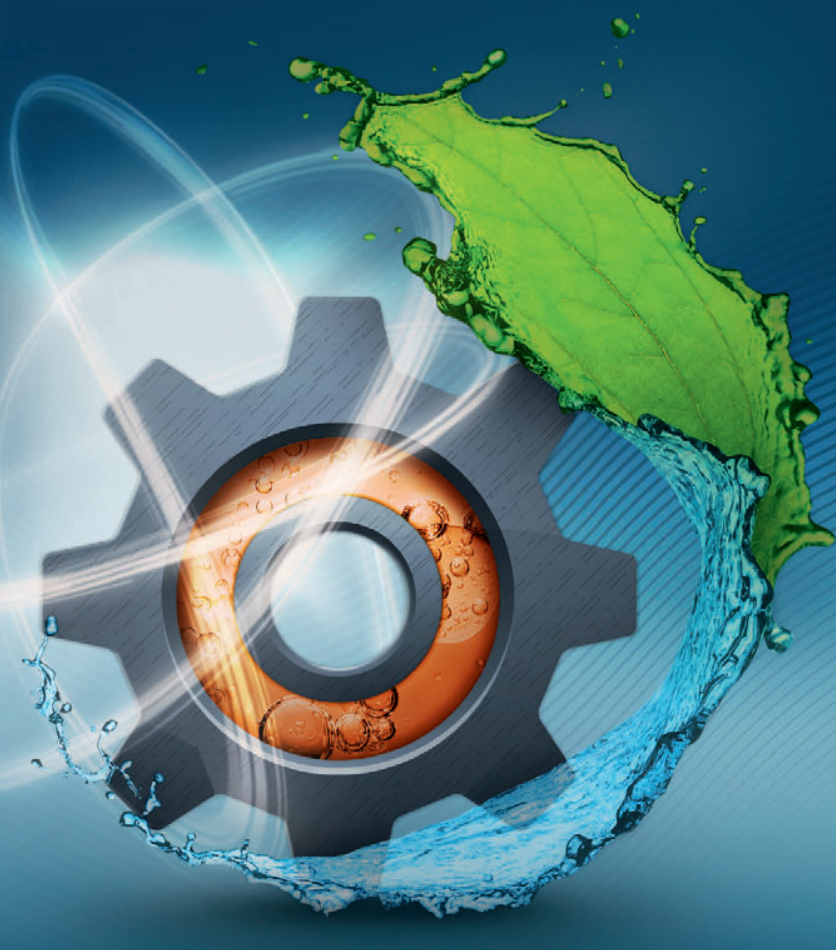

BE INFORMED.

BE INSPIRED. BE THERE.

> World Forum and Leading Show

for the Process Industries

> 3,800 Exhibitors from 50 Countries

> 170,000 Attendees from 100 Countries

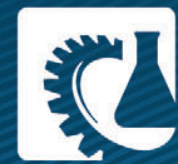

Wwwrachemarde 